\title{
Crural hypertrophy associated with centronuclear myopathy
}

\author{
P. B I L L, G. C O LE, N.S. F. PR OCT OR, D. SAFFER, \\ A N D A. B O T E S
}

From the Department of Neurology, Baragwanath Hospital, Transvaal, and Neuropathology and Electron Microscopy Units, Department of Anatomical Pathology, School of Pathology of the University of the Witwatersrand, and South African Institute for Medical Research, Johannesburg, South Africa

SUMMARY A case of centronuclear myopathy of adult onset with striking hypertrophy of lower limb muscles in a black South African man is described with details of the light microscopic, histochemical, and ultrastructural features. The association of hypertrophied muscles with centronuclear myopathy has not to our knowledge been reported previously and it is felt that this may be a variant of the condition.

Centronuclear myopathy-initially termed myotubular myopathy-is a relatively rare congenital myopathy of uncertain pathogenesis. Reported cases have shown a wide spectrum of clinical features. The occurrence of oculofacial palsy with diffuse proximal limb weakness and areflexia has been noted in many patients. Other cases have presented at birth with hypotonia and subsequent respiratory difficulty, and do not usually survive. An onset in later life with a nonspecific myopathic picture is also recognised.

\section{Case report}

The patient is a black male aged 60 years who was admitted to Baragwanath Hospital in 1978 with symptoms of poor vision, polyuria, and lassitude. A blood sugar determination of $55 \mathrm{mmol} / 1$ confirmed the diagnosis of diabetes mellitus which was controlled with insulin injections. Since childhood he had been aware of hypertrophy of the leg and thigh muscles. There were no symptoms to suggest muscular weakness in childhood or early adulthood. In recent years he noticed difficulty in walking and climbing stairs, and was unable to run. There was no history of cramps, joint pains, or Raynaud's phenomenon. His father was said to have had enlarged muscles of the lower limbs but was not disabled and died in old age. One brother

Address for reprint requests: Dr G. Cole, Department of Neuropathology, South African Institute for Medical Research, PO Box 1038, Johannesburg 2000, South Africa.

Accepted 6 December 1978 has hypertrophy of the calf muscles which has been present since childhood.

Examination revealed a mildly obese middleaged black male. His blood pressure was $130 / 90$ $\mathrm{mmHg}$, pulse $96 /$ minute, and there were no abnormal findings on systematic examination. A right homonymous hemianopia was present and ascribed to vascular complications associated with diabetes mellitus. There was no weakness of the facial, bulbar, masticatory, or neck muscles. Ocular movement was normal. There was moderate wasting of the deltoid, biceps, triceps, brachioradialis, and forearm extensor muscles. The most prominent weakness in the upper limbs involved the biceps, triceps, and shoulder abductors with lesser weakness of the flexor and extensor muscles of the forearm. In the lower limbs there was marked hypertrophy of the calf muscles, tibialis anterior and, to a lesser extent, the anterior thigh muscles (Fig. 1). Pelvic girdle weakness resulted in a slow waddling gait. Weakness was also present in the extensors and evertors of the feet, but the calf muscles were of normal strength. There was no fasciculation or myotonia. Tendon reflexes were absent but there were normal plantar responses. There was a loss of vibration sense over the feet. Stepping onto a low stool was performed with difficulty, and he was unable to rise from a squatting position.

INVESTIGATIONS

Full blood count, plasma urea, electrolytes, and thyroid function were normal. The level of serum 


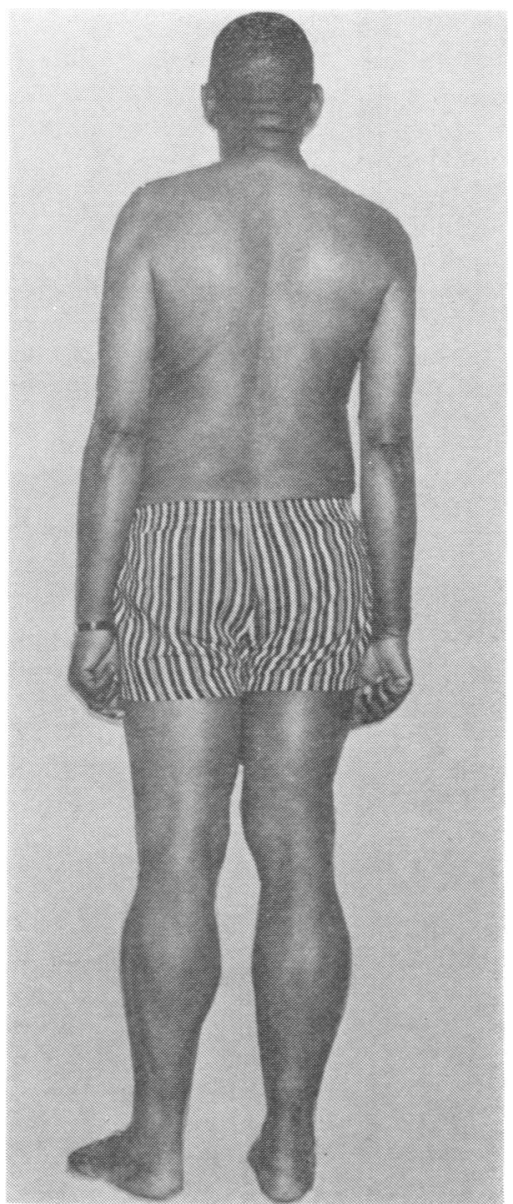

Fig. 1 The patient. Hypertrophy of calf muscles is evident.

creatine phosphokinase (CPK) was raised (332 iu/1), while the serum glutamic oxaloacetic transaminase (SGOT), serum glutamic pyruvate transaminase (SGPT), and lactic dehydrogenase (LDH) levels were normal. An electromyogram of the biceps, quadriceps, and tibialis anterior showed numerous low voltage, highly polyphasic motor unit poientials, and large simple motor unit potentials of up to 7 millivolts amplitude. No fibrillation potentials were present. Motor conduction velocity in the peroneal nerve was 55 metres/second. A muscle biopsy was taken from the right biceps and quadriceps muscle in order to sample an atrophic and an hypertrophied muscle.

\section{FAMILY HISTORY}

The father, who died in old age, was said to have had hypertrophy of the calf muscles. The patient

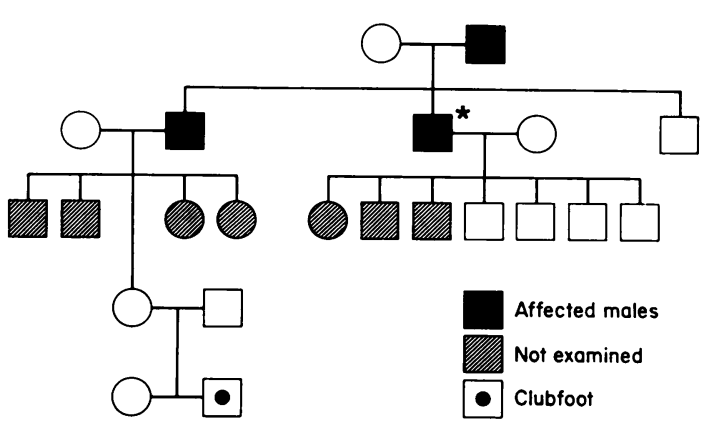

Fig. 2 Pedigree of patient.

has two brothers (see Fig. 2) but only one was available for examination, and he had asymmetrical hypertrophy of the anterior tibial and calf muscles with the left being larger than the right. There was no weakness in the lower limbs. The upper limbs showed no hypertrophy but mild weakness of the shoulder abductors, biceps, and triceps. Reflexes were normal as were serum CPK and aldolase levels. He refused further investigation. The patient has six sons and one daughter, all of whom are reported to be normal. Examination of four of the children revealed no abnormality, and CPK estimations were normal. None of the children of the brother were available for examination, but all are said to be normal. A grandson has a clubfoot, but normal serum CPK was found.

\section{HISTOPATHOLOGY}

Muscle biopsy was taken from the right biceps and quadriceps muscle. Specimens from each were prepared for paraffin embedding, histochemical examination, and electron microscopic examination.

The paraffin-embedded material was cut at $4 \mu \mathrm{m}$. The stains employed were haematoxylin and eosin (H and E), periodic acid Schiff (PAS), phosphotungstic acid haematoxylin (PTAH), and Masson's trichrome and modified Gomori's trichrome. The biopsy specimens from biceps and quadriceps showed virtually the same pathological changes. Longitudinal and transverse sections were examined. There was a marked increase in perimyseal fat, and this was more prominent in the sections from the quadriceps (hypertrophied muscle). There were many very small, rounded fibres, most of which contained one or more central nuclei. The average diameter of the small fibres was $16 \mu \mathrm{m}$. In a number of these fibres the nuclei were clumped together resulting in a large 

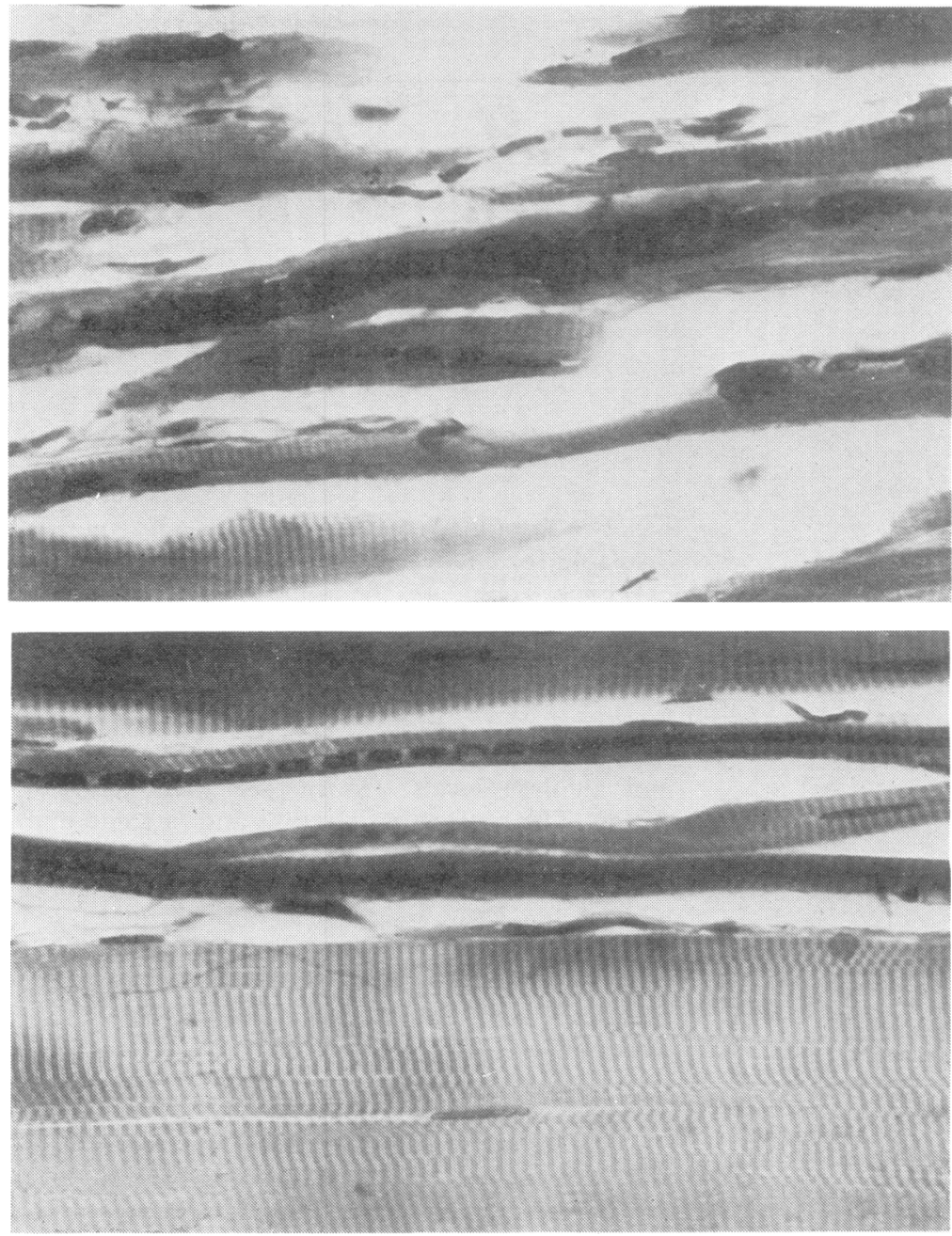

Fig. 3 Longitudinal section of muscle showing marked clumping of central nuclei. $H$ and $E$, original magnification $\times 400$.

Fig. 4 Longitudinal section of muscle showing chains of central nuclei in markedly atrophic fibres. $H$ and $E$, original magnification $\times 400$. basophilic and usually central mass (Fig. 3). A moderate number of hypertroph:ed fibres was present with an average diameter of $62 \mu \mathrm{m}$. The hypertrophied fibres tended to be in groups of two or three, and contained several central and peripheral nuclei, but no clumping of nuclei as described in the atrophic fibres. Chains of centrally placed nuclei were well demonstrated in the longitudinal sections (Fig. 4). An occasional area of phagocytosis with an inflammatory cell reaction was present. In one such area an unusual feature was the presence of a mitotic figure in a muscle fibre.

\section{HISTOCHEMISTRY}

Histochemical stains employed were the routine ATPase reactions at $\mathrm{pH} 9.4$, with preincubation at
$\mathrm{pH}$ of 4.6 and 4.3 , phosphorylase reaction, and the NADH-TR oxidative stain. The methods followed were those described by Dubowitz and Brooke (1973). There was extremely poor differentiation on ATPase reactions, and the fibre types could not be identified. On the NADH-TR and phosphorylase reactions, however, differentiation was possible, and the small atrophic fibres were both type 1 and 2 , while the hypertrophied fibres were predominantly type 2 . On the NADH-TR stain (Fig. 5) some of the atrophic fibres showed dense staining and no architectural features could be appreciated. Other fibres showed scattered clumping of fibrils and increased uptake of the stain. The hypertrophied fibres showed no obvious architectural abnormality. A lack of staining in the centre of some of the fibres was attributed to artefact. 

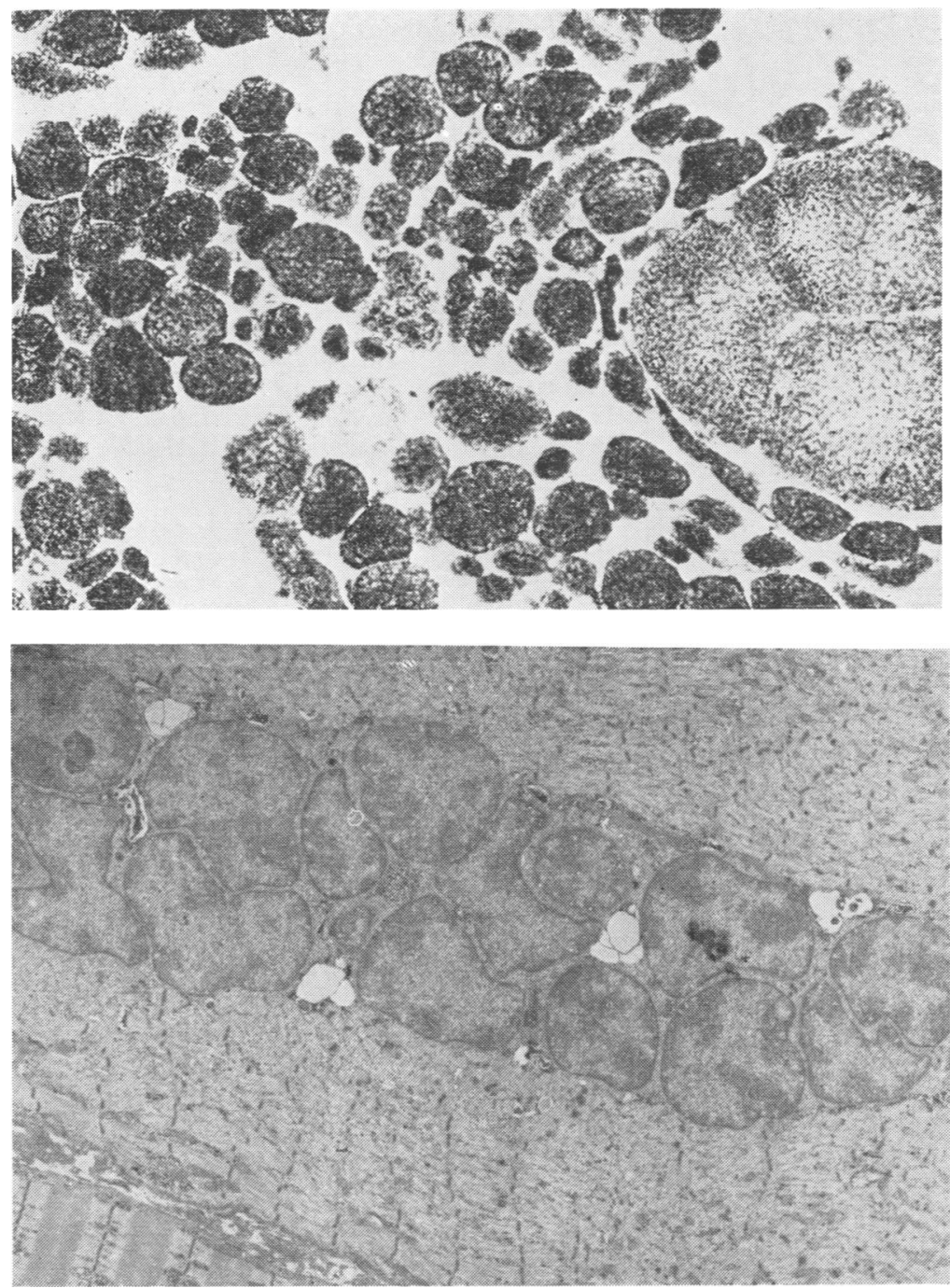

Fig. 5 Illustrating the presence of type 1 and type 2 fibres. The hypertrophied fibres are predominantly type 2 . Increased uptake of stain obliterates the architectural features of some of the type 1 fibres. NADH-TR, original magnification $\times 160$.
Fig. 6 Electronmicrograph. Central nuclear chain two to three nuclei deep. There is marked variation in size and distorted nuclear outlines. Note destruction of normal myofibrillar pattern. Original magnification $\times 6000$.

\section{ELECTRONMICROSCOPY}

The muscle was fixed in $2.5 \%$ glutaraldehyde in cacodylate buffer and post-fixed in osmium tetroxide.

Nuclear chains were prominent, and consisted of rows of either single or multiple nuclei. The morphology and nuclear outline were usually normal in the fibres with chains of nuclei in single file. Other fibres contained nuclear chains two to three nuclei deep with grossly distorted nuclear outlines fitting together in an intricate pattern, and a marked variation in nuclear size was present (Fig. 6). The chains of nuclei were either centrally or eccentrically placed, and were often in close approximation to the sarcolemma. Glycogen par- ticles were present between the nuclei. In occasional areas fragments of myofibrils with recognisable $\mathrm{Z}$ band material could be identified between the nuclei. Nuclear chains were for the most part closely approximated to the myofibrils, but some were separated from them by accumulations of glycogen and mitochondria. In one particular fibre two central chains were present and separated by several normal appearing myofibrils. Nuclear chains were seen both in fibres which were grossly abnormal with destruction of normal myofibrillar pattern, and in normal appearing fibres. Conversely, fibres with grossly abnormal architecture were often seen without central nuclei. Hypertrophied fibres were not observed to contain 

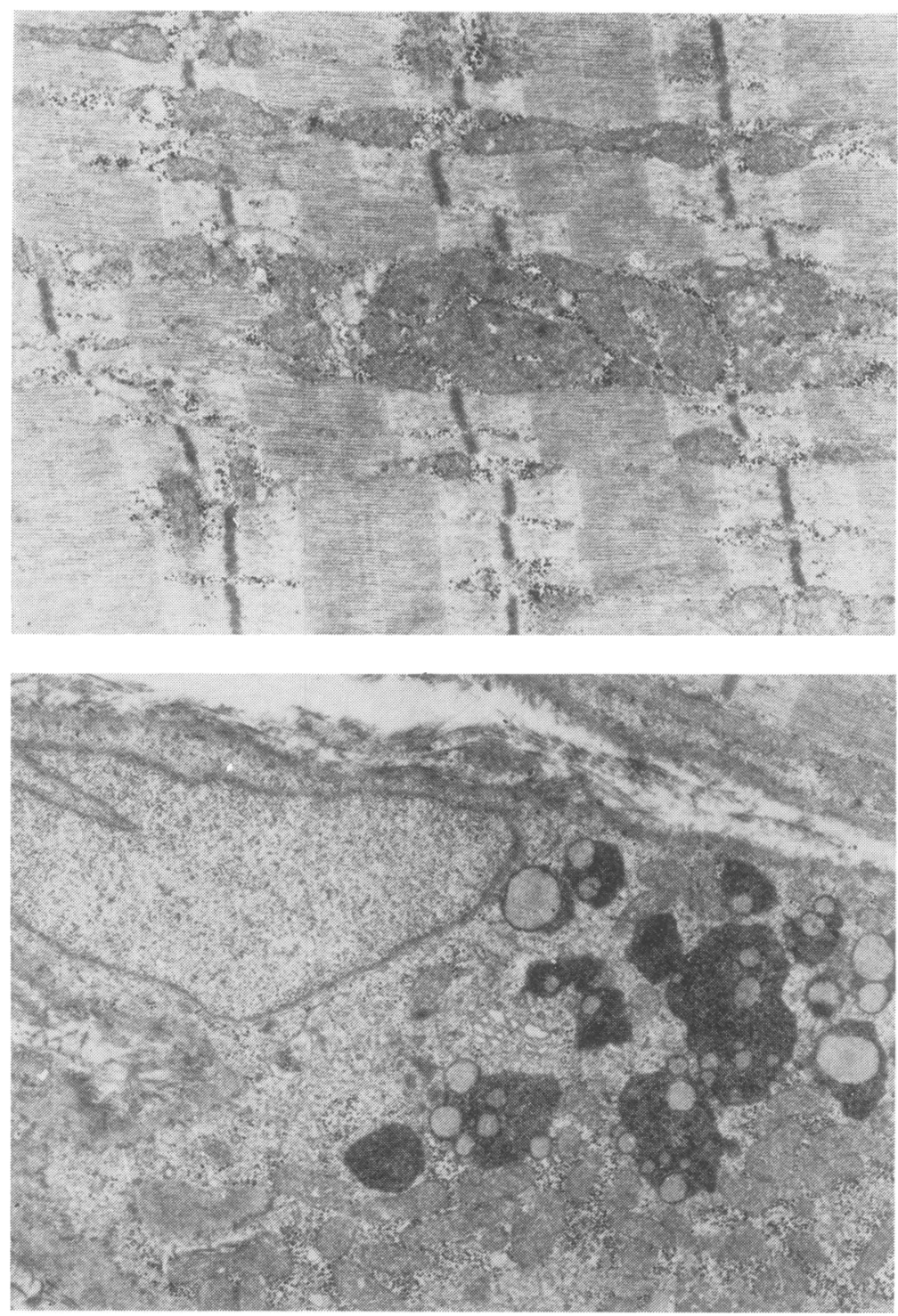

Fig. 7 Electronmicrograph. Accumulations of markedly enlarged mitochondria extending the length of several sarcomeres. Original magnification $\times 20800$.

Fig. 8 Electronmicrograph. Accumulations of lysosomes, mitochondria, and glycogen in the subsarcolemmal space. Original magnification $\times 18700$. nuclear chains, although central and subsarcolemmal nuclei were usually present. Focal changes were seen in many otherwise normal fibres. These consisted of $\mathrm{Z}$ band streaming and disruption and myofibrillar disruption. In such areas mitochondria were scanty or absent. Some fibres contained accumulations of markedly enlarged mitochondria in the central region extending the length of several sarcomeres (Fig. 7). The internal appearance of these large mitochondria appeared normal. Another frequent finding in fibres with subsarcolemmal nuclei was accumulations of lysosomes, mitochondria, and glycogen in the subsarcolemmal space adjacent to the nucleus (Fig. 8). Damaged fibres showed such severe fragmentation of myofibrils that A and I bands could not be discerned. The $\mathrm{Z}$ band material was fragmented and irregularly scattered. Mitochondria of normal appearance could be found but sarcoplasmic triads were not seen. In less severely damaged fibres excess intermyofibrillar glycogen was present. No abnormal changes were found in the sarcolemma or blood vessels. 


\section{Discussion}

The clinical features and genetic aspects of centronuclear myopathy are discussed by Bill et al. (1979). Although most cases are seen in childhood and infancy, the condition has been described in adults (Harriman and Haleem, 1972; McLeod et al., 1972). In none of the cases has hypertrophy been described as a feature.

Several striking features on light microscopy are of considerable interest. The large clumps of nuclei in the central areas were present in both atrophic (biceps) and hypertrophic (quadriceps) muscle, but this phenomenon was confined to atrophic muscle fibres. Equally frequent were rows of single nuclei. The marked increase in perimysial fat is the likely cause of the hypertrophy, and on light microscopy the amount of fat was greatest in the biopsy sample from the quadriceps which clinically was hypertrophied. In the biceps muscle the increase of fat was less prominent, corresponding with lack of hypertrophy in the upper arm muscles. The overall pattern is suggestive of a neurogenic disorder in that there is large group atrophy with some fascicles containing groups of hypertrophic fibres. The probability that centronuclear myopathy is a neurogenic disorder should, therefore, be considered and in this respect, the recent description of hypertrophy of calf muscles in spinal muscle atrophy is of considerable interest (Pearn and Hudgson, 1978). It should also be noted that fibrillation potentials have been found in some of the reported cases of centronuclear myopathy.

On histochemistry, poor differentiation with the ATPase stains is well recognised (van Wijngaarden et al., 1969; Harriman and Haleem, 1972; Headington et al., 1975). This had led to the suggestion that the fibres are undifferentiated, or predomin- antly of the type 1 variety. However, fibre type differentiation is present on NAD stains.

Electronmicroscopy has revealed that central nuclear chains occur equally in fibres with relatively normal architecture and in fibres with gross myofibrillar disruption. The juxtaposition of nuclei with myofibrils also indicates that the perinuclear halo seen in formalin fixed tissue is probably the result of an artefact. In general, however, the electronmicroscopic appearances have not helped in elucidating the pathogenesis of this condition. In considering the genetic aspects no definite conclusion can be reached in regard to this case. Although the patient's brother is probably affected we do not have biopsy evidence. The father was reported to have large muscles but is dead. None of the children are clinically affected.

\section{References}

Bill, P., Cole, G., and Proctor, N. S. F. (1979). Centronuclear myopathy. Journal of Neurology, Neurosurgery, and Psychiatry, 42, 548-556.

Dubowitz, V., and Brooke, H. (1973). Muscle Biopsy: A Modern A pproach. W. B. Saunders: London.

Harriman, D. G. F., and Haleem, M. A. (1972). Centronuclear myopathy in old age. Journal of Pathology, 108, 237-248.

Headington, J. T., McNamara, J. O., and Keith Brownell, A. (1975). Centronuclear myopathy: histochemistry and electronmicroscopy. Report of 2 cases. Archives of Pathology, 99, 16-24.

McLeod, J. G., Baker, W. de C., Lethlean, A. K., and Shorey, C. D. (1972). Centronuclear myopathy with autosomal dominant inheritance. Journal of the Neurological Sciences, 15, 375-388.

Pearn, J., and Hudgson, P. (1978). Anterior horn cell degeneration and gross calf hypertrophy with adolescent onset. Lancet, 1, 1059-1061.

van Wijngaarden, G. K., Fleury, P., Bethlem, J., and Hugo Meijer, A. E. F. (1969). Familial myotubular myopathy. Neurology (Minneapolis), 19, 901-908. 